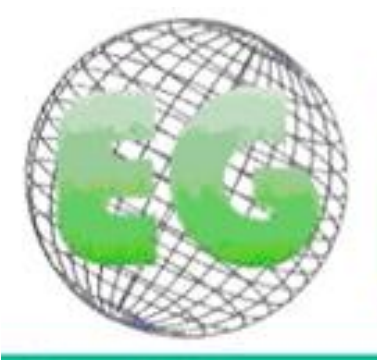

ISSN $1695-6141$
$\mathbf{N}^{\circ} 35$

\title{
REVISIONES
}

\section{Teoría del manejo de síntomas desagradables para el cuidado de enfermería en cirugía}

Management theory of unpleasant symptoms nursing care in surgery

\section{*Laguado Jaimes, Elveny **Gómez Díaz, Martha Patricia}

\author{
"Maestría en Enfermería. Docente Programa de Enfermerìa. E-mail: \\ elveny.laguado@campusucc.edu.co ${ }^{* *}$ Enfermera. Docente Programa de Enfermeria. Universidad \\ Cooperativa de Colombia. Sede Bucaramanga.
}

Palabras clave: cuidado posoperatorio; síntomas desagradables; experiencia de los sintomas,

Keywords: postoperative care; unpleasant symptoms; experience symptoms.

\section{RESUMEN}

Mediante la revisión sistemática de artículos relacionados con los cuidados de enfermeria en pacientes sometidos a procesos quirúrgicos, se clasifican por niveles de evidencia; con base en la teoria de sintomas desagradables de Elizabeth Lenz en la que mediante sus tres componentes: síntomas, factores influyentes y resultados de desempeño y además cuatro dimensiones: la angustia, la calidad, la duración y la intensidad de los síntomas, se argumenta la importancia de los cuidados de enfermería basado en la teoría de rango medio: síntomas desagradables de Elizabeth Lenz y colaboradores, para ser aplicada en el contexto del cuidado de pacientes en proceso de intervención quirúrgica, de tal forma que disminuyan los efectos de los sìntomas en el posoperatorio.

\section{ABSTRACT}

Through systematic review related to nursing care for patients undergoing surgical procedures articles, classified by levels of evidence; based on the theory of unpleasant symptoms Elizabeth Lenz in which by its three components: symptoms, influencing factors and performance outcomes while four dimensions: anxiety, quality, duration and intensity of symptoms, argue the importance of nursing care based on the mid-range theory: unpleasant symptoms of Elizabeth Lenz et al, to be applied in the context of the care of patients undergoing surgical intervention, so as to decrease the effects of symptoms in the postoperatively. 


\section{INTRODUCCIÓN}

En la teoría de síntomas desagradables de Elizabeth Lenz y colaboradores, desarrollada en diferentes situaciones de salud de los pacientes, su aplicación integra la información con el propósito de mejorar la experiencia de la sintomatología, información útil para diseñar métodos efectivos de prevención y mejorar sus efectos negativos.

Está conformada por tres componentes importantes: síntomas, factores influyentes y resultados de desempeño y además cuatro dimensiones: la angustia, la calidad, la duración y la intensidad de los síntomas.

Para la comprensión de la teoría, los síntomas los expresa como aquellos que son experimentados por el individuo y definidos como los indicadores del cambio, percibidos en el funcionamiento normal; los factores influyentes son fisiológicos, sicológicos, situacionales, influyen en la percepción de los síntomas, en la realización o el resultado de la experiencia del síntoma, el impacto en las actividades cognitivas y funcionales.

Además cuenta con cuatro dimensiones, la angustia atributo que se refiere al grado en el cual la persona es molestada por la experiencia del síntoma; la calidad es la coordinación existente entre la aparición de un síntoma y una actividad específica; la duración incluye la frecuencia con la que un síntoma ocurre; la intensidad observa la gravedad, la fuerza o la cantidad del síntoma que se experimenta

En esta revisión se parte de la búsqueda bibliográfica en bases datos: Scielo, Lilacs, Proquest, Ebrary entre otras; para clasificar la información, las palabras clave son las etapas del proceso quirúrgico denominadas: fase preoperatoria y posoperatorio inmediato en la población adulta y para determinar su pertinencia y aporte al desarrollo del tema se valora de acuerdo a los niveles de evidencia de los artículos, para lo cual se utiliza la clasificación de la United States Preventive Task Force (USPSTF) ${ }^{(1)}$, que clasifica las evidencias en 3 niveles, como se muestra en la Tabla 1.

Los artículos presentados a continuación, argumentan la importancia de los cuidados de enfermería basado en la teoría de rango medio: síntomas desagradables de Elizabeth Lenz y colaboradores, para una atención con calidad. Además hay que tener en cuenta que el cuidado holístico implica el abordaje de las dimensiones física, psicológica y situacional. 


\begin{tabular}{|c|l|}
\hline \multicolumn{2}{|c|}{ Tabla I. Jerarquía de los estudios por el tipo de diseño según US } \\
PreventiveTaskForce
\end{tabular}

Fuente: United States Preventive Task Force (USPSTF)

\section{NIVELES DE EVIDENCIA SEGÚN LOS ARTICULOS REVISADOS}

\begin{tabular}{|c|c|c|}
\hline ESTUDIO & FUENTE & $\begin{array}{l}\text { NIVEL DE } \\
\text { EVIDENCIA }\end{array}$ \\
\hline $\begin{array}{l}\text { Cuidados de enfermería } \\
\text { realizados en el paciente } \\
\text { quirúrgico en el período } \\
\text { preoperatorio- Berlina E y } \\
\text { col (2009) }\end{array}$ & $\begin{array}{l}\text { Scielo } \\
\text { Revista da Escola de } \\
\text { Enfermagem da USP } \\
\text { Online: } \\
\text { http://dx.doi.org/10.1590/S0080- } \\
62342009000100002\end{array}$ & III \\
\hline $\begin{array}{l}\text { Comunicación terapéutica en } \\
\text { la atención pre-operatoria de } \\
\text { mastectomía; Lavina Santos } \\
\text { y col (2010) }\end{array}$ & $\begin{array}{l}\text { Scielo } \\
\text { Revista Brasilera de enfermage } \\
\text { Online: } \\
\text { http://dx.doi.org/10.1590/S0034- } \\
71672010000400027\end{array}$ & II-1 \\
\hline $\begin{array}{l}\text { Cuidados de enfermería a } \\
\text { usuarios hospitalizados de la } \\
\text { Unidad Clínica de Cirugía en } \\
\text { la fase preoperatoria del } \\
\text { Hospital Uyapar de Puerto } \\
\text { Ordaz Estado Bolívar primer } \\
\text { semestre 2005;Doris Walter } \\
\text { (2011) }\end{array}$ & $\begin{array}{l}\text { Lilacs } \\
\text { Online: } \\
\text { http://saber.ucv.ve/123456789/3 } \\
\underline{69}\end{array}$ & III \\
\hline $\begin{array}{l}\text { Cuidados preoperatorios de } \\
\text { mastectomía bajo la óptica } \\
\text { de la mujer; Oliveira y col } \\
(2005)\end{array}$ & $\begin{array}{l}\text { Scielo } \\
\text { Revista cubana de enfermería } \\
\text { Online: } \\
\text { http://scielo.sld.cu/scielo.php?sc } \\
\text { ript=sci_arttext\&pid=s0864- } \\
\text { 03192005000200011> }\end{array}$ & II-2 \\
\hline
\end{tabular}




\begin{tabular}{|c|c|c|}
\hline $\begin{array}{l}\text { Cuidados peri operatorios } \\
\text { del paciente quirúrgico; } \\
\text { Echeverri Gustavo y col } \\
\text { (2002) }\end{array}$ & $\begin{array}{l}\text { Atención integral de enfermería } \\
\text { al adulto y anciano con lesiones } \\
\text { traumáticas y quirúrgicas } \\
\text { Online: } \\
\text { http://objetos.univalle.edu.co/?q } \\
=\text { node/368 }\end{array}$ & II-1 \\
\hline $\begin{array}{l}\text { Enfermería } \quad \text { quirúrgica; } \\
\text { Chames de Fuentes (2008) }\end{array}$ & $\begin{array}{l}\text { ProQuest } \\
\text { www.proquest.com/ } \\
\text { Online: } \\
\text { http://www.fm.unt.edu.ar/carrera } \\
\underline{\text { s/webenfermeria/documentos/E }} \\
\underline{\text { nfermeria Quirurgica Modulo } 0} \\
\end{array}$ & II-1 \\
\hline $\begin{array}{l}\text { Estandarización de los } \\
\text { cuidados de enfermería en } \\
\text { Preanestesia; } \\
\text { Andreu Atik P y col (2012) }\end{array}$ & $\begin{array}{l}\text { Scielo } \\
\text { Online: } \\
\text { http://hispasante.hispagenda.co } \\
\text { m/documentacion/guias/enferm } \\
\text { eria/Estandarizacion cuidados } \\
\text { enfermeria Preanestesia.pdf }\end{array}$ & ||$-1$ \\
\hline $\begin{array}{l}\text { Cirugía: entre la angustia y } \\
\text { la alegría a la vez; Salazar } \\
\text { AngelaMaria (2011) }\end{array}$ & $\begin{array}{l}\text { Redalyc } \\
\text { Online: } \\
\text { http://aquichan.unisabana.edu.c } \\
\text { o/index.php/aquichan/article/vie } \\
\text { w/1900/2476>. }\end{array}$ & III \\
\hline $\begin{array}{l}\text { Ansiedad y Cirugía. } \\
\text { Repercusiones en el } \\
\text { paciente sometido a cirugía; } \\
\text { Barrilero Gómez Jose y col } \\
(1996)\end{array}$ & $\begin{array}{l}\text { Scielo } \\
\text { Diplomado en Enfermería } \\
\text { (1996) } \\
\text { Online: } \\
\text { http://www.uclm.es/ab/enfermeri } \\
\text { a/revista/numero\%208/ansicirug } \\
\text {.htm }\end{array}$ & III \\
\hline $\begin{array}{l}\text { Influencia de la ansiedad } \\
\text { prequirúrgica en la } \\
\text { evolución de la cirugía de } \\
\text { las várices; PerezDiaz } \\
\text { Raquel y col (1997) }\end{array}$ & $\begin{array}{l}\text { Redalyc } \\
\text { Rev Cubana Angiol y CirVasc } \\
\text { Online: } \\
\text { http://bvs.sld.cu/revistas/ang/vol } \\
1200 / \text { ang02200.htm } \\
\end{array}$ & III \\
\hline $\begin{array}{l}\text { Histerectomía y ansiedad: } \\
\text { estudio comparativo entre } \\
\text { dos tipos de preparación } \\
\text { psicológica pre quirúrgica; } \\
\text { Cárdenas Andrea y col } \\
(2005)\end{array}$ & $\begin{array}{l}\text { Redalyc } \\
\text { Revista Colombiana de } \\
\text { Obstetricia y Ginecología, vol. } \\
56 \text { numero } 3 \\
\text { Online: } \\
\text { http://redalyc.uaemex.mx/redaly } \\
\text { c/pdf/1952/195214311003.pdf }\end{array}$ & III \\
\hline $\begin{array}{l}\text { Ansiedad preoperatoria en el } \\
\text { Hospital General de México/ } \\
\text { Preoperativeanxiety in the }\end{array}$ & $\begin{array}{l}\text { Online: } \\
\text { http://www.medigraphic.com/pdf }\end{array}$ & III \\
\hline
\end{tabular}




\begin{tabular}{|c|c|c|}
\hline $\begin{array}{l}\text { Hospital General de México; } \\
\text { Ruiz López Enrique (2000) }\end{array}$ & s/h-gral/hg-2000/hg004b.pdf & \\
\hline $\begin{array}{l}\text { ARTICULO: Efecto de una } \\
\text { visita pre quirúrgica de } \\
\text { enfermería perioperatoria } \\
\text { sobre la ansiedad y el dolor; } \\
\text { Chirveches Emilia (2006) }\end{array}$ & $\begin{array}{l}\text { Scielo } \\
\text { Online: } \\
\text { http://www.elsevier.es/es/revista } \\
\text { s/enfermeria-clinica-35/efecto- } \\
\text { una-visita-prequirurgica- } \\
\text { enfermeria-perioperatoria- } \\
\text { ansiedad-13084268-originales- } \\
\underline{2006}\end{array}$ & I \\
\hline $\begin{array}{l}\text { Pre-operative education for } \\
\text { hip or knee replacement; } \\
\text { McDonald Steve y col (2003) }\end{array}$ & $\begin{array}{l}\text { Library } \\
\text { The Cochrane Library número } 2 \text {, } \\
2003\end{array}$ & II-3 \\
\hline $\begin{array}{l}\text { Enseñanza pre quirúrgica y } \\
\text { disminución de la ansiedad } \\
\text { en el control del temor en } \\
\text { pacientes programados para }\end{array}$ & $\begin{array}{l}\text { Scribd } \\
\text { es.scribd.com/.../Tecnicas- } \\
\text { Basicas-de-Enfermeria-Medico- } \\
\text { Quirurgica }\end{array}$ & II -2 \\
\hline $\begin{array}{l}\text { cirugía; Sepulveda Martha y } \\
\text { col (2004) }\end{array}$ & $\begin{array}{l}\text { Tesis Especialización en } \\
\text { atención de Enfermería en } \\
\text { Quirófanos y Central de } \\
\text { Esterilización. } \\
\text { Industrial de Universidad } \\
\text { Facultad de salud }\end{array}$ & \\
\hline $\begin{array}{l}\text { Aplicación de la teoría de los } \\
\text { síntomas desagradables en } \\
\text { el manejo del dolor. } \\
\text { Investigación en Enfermería; } \\
\text { Archury Diana Marcela } \\
\text { (2007) }\end{array}$ & $\begin{array}{l}\text { REDALYC } \\
\text { PONTIFICIA UNIVERSIDAD } \\
\text { JAVERIANA -COLOMBIA } \\
\text { ONLINE: } \\
\text { HTTP://REDALYC.UAEMEX.MX } \\
\text { /PDF/1452/145212858002.PDF } \\
\text { FECHA DE ACCESO 14 MARZ. } \\
\text { 2012. }\end{array}$ & II-1 \\
\hline $\begin{array}{ll}\text { "stress" } & \text { preoperatoria; } \\
\text { PerezMencia } & \text { Ma. Teresa y } \\
\text { col (2005) } & \end{array}$ & $\begin{array}{l}\text { Ebrary } \\
\text { Universidad Complutense de } \\
\text { Madrid. } \\
\text { Online: } \\
\text { http://site.ebrary.com/lib/ucoope } \\
\text { rativasp/Doc?id=10088239\&ppg } \\
=68 \\
\text { Copyright } \odot 2005 \text {. Universidad } \\
\text { Complutense de Madrid. } \\
\text { Allrightsreserved. }\end{array}$ & III \\
\hline
\end{tabular}

Fuente: Elaboración propia

En la revisión bibliográfica los estudios refieren los efectos que ocasiona en los pacientes a nivel físico, sicológico, cuando son sometidos a procesos quirúrgicos. 
Este tipo de pacientes, suelen experimentar distintos miedos y esperanzas, por lo tanto el personal de enfermería debe determinar las percepciones, emociones, conductas y sistemas de apoyo que pueden ayudar o interferir en la capacidad del paciente para progresar durante el período quirúrgico ${ }^{(2)}$.

A nivel internacional, en Barcelona (España) Chirveches, Emilia y col ${ }^{(3)}$ plantearon en el estudio el efecto de una visita prequirúrgica de enfermería perioperatoria sobre la ansiedad y el dolor, mediante un ensayo clínico controlado. Uno de los grupos recibía información adicional sobre el proceso quirúrgico mediante una visita prequirúrgica estructurada de enfermería, el otro grupo de pacientes de control recibía la atención prequirúrgica habitual. Los resultados muestran estadísticamente la ansiedad situacional en el grupo intervención respecto al grupo control y un aumento de la satisfacción de los pacientes del grupo intervención respecto al proceso quirúrgico.

Otro estudio donde se manifiesta la ansiedad perioperatoria es el de Pérez Díaz y col. - Cuba ${ }^{(4)}$, el nivel de ansiedad prequirúrgica evidenciada en la recuperación posoperatoria fue medida mediante la escala Ansiedad Rasgo - Estado, de Ch. Spielberger, la ansiedad que presentan los pacientes a medida que se acerca la intervención quirúrgica y alcanza su máxima intensidad, el día de la operación; manifiestan síntomas psicofisiológicos que usualmente acompañan la ansiedad durante los momentos del acto operatorio, en ocasiones pueden persistir hasta muchos meses después de la intervención quirúrgica; incluyen taquicardias, arritmias, elevaciones de la tensión arterial, disnea, etc.; finalmente asocian la ansiedad preoperatoria con mayor frecuencia de accidentes anestésicos, vulnerabilidad a las infecciones, mayor estancia hospitalaria y mayores niveles de dolor posoperatorio.

El estudio de Chávez Nancy (5) "Efectividad de un programa educativo en el incremento del nivel de conocimiento que tiene el paciente pre-quirúrgico en el Hospital II de Lima-Perú" estudio de tipo Cuantitativo, nivel aplicativo, método cuasiexperimental, la muestra fue de 30 pacientes pre-quirúrgicos, los datos fueron recolectados mediante una entrevista, utilizando un cuestionario individualizado en los consultorios de anestesiología, luego se aplicó el programa educativo en forma individualizada; dichos pacientes fueron citados después de una semana y aplicándose el cuestionario para verificar la eficacia de dicho programa. Antes de la aplicación del programa educativo se evidencia en los pacientes pre-quirúrgicos un conocimiento medio referente su cirugía, anestesia y preparación física para la misma. Luego de la aplicación se incrementóa conocimiento alto y disminución en el conocimiento medio y bajo; concluyen que el programa educativo fue eficaz, lo cual favorece a los pacientes pre-quirúrgicos a enfrentar situaciones durante el proceso de su cirugía.

Entre los estudios encontrados en relación a la aplicación de la teoría de los síntomas desagradables en el manejo del dolor, está el de Achury Saldaña Diana M. ${ }^{(6)}$ Este estudio resalta el síntoma desagradable, analiza la necesidad de aplicar en nuestra práctica diaria la teoría de los síntomas desagradables como una herramienta en la evaluación del dolor y para entender el verdadero significado del dolor desde un punto de vista integral, conociendo las características propias de cada individuo, al igual que sus factores fisiológicos, psicológicos y ambientales. En el estudio revisaron conceptos generales sobre el dolor y teorías de mediano rango aplicando la teoría de síntomas desagradables en la experiencia y vivencia propia de la percepción del dolor 
agudo y crónico, la enfermera y el enfermero realizan un análisis integral de los síntomas y su impacto en los entornos social, cultural y emocional, y pueda establecer intervenciones de cuidado individuales y no generalizada; por lo anterior en pacientes que van a procesos quirúrgicos es un síntoma que es frecuentemente manifestado y el cual experimenta el paciente en diferentes escalas según su percepción y conocimiento.

Otros estudios relacionados con el cuidado de enfermería en cirugía, Berendina ${ }^{(7)}$, concluye la poca orientación en los procedimientos quirúrgicos y los cuidados de enfermería; esto contribuye al debate sobre la necesidad de un cambio en la práctica de la enfermería en el hospital, para brindar atención con calidad en los servicios especialmente en área quirúrgica. Igualmente el doctor Heberto R. Priego Álvarez ${ }^{(8)}$ en un estudio realizado en el Centro Médico ISSET a un grupo de enfermeras quirúrgicas y generales observa y describe la calidad en las visitas preoperatorias, concluyendo que las enfermeras quirúrgicas no cumplen en su totalidad con las funciones, a pesar de tener los conocimientos técnicos y científicos para realizar dicha intervención, las enfermeras generales son quienes realizan la mayoría de las visitas pre operatorias, esto repercute en la calidad técnica y humana de las acciones de las enfermeras quirúrgicas y por ende de la institución.

Otro aspecto de gran importancia, es la comunicación terapéutica como indica Lavinas Santos ${ }^{(9)}$ los pacientes pueden aprender, entender y buscar una solución a su malestar a través de la construcción y la reestructuración de la información lo que contribuye a la interacción con el personal de salud, lo que permite entender al paciente y la forma en que ve, siente y percibe y actúa en el mundo.

En los procesos quirúrgicos, dependiendo de la forma como reaccione el paciente, su capacidad de afrontamiento favorece su recuperación; es así como en el estudio de McDonald S, Hetrick $S^{(10)}$ se tomó una muestra de 782 participantes realizada en Australia ante el reemplazo de rodilla o cadera, procedimiento quirúrgico importante que puede ser física y psicológicamente estresante para los pacientes. Existe una hipótesis según la cual la educación pre quirúrgica reduce la ansiedad y mejora los resultados postoperatorios. En el estudio concluyen que hay pocas pruebas que apoyen el uso de educación pre quirúrgica por encima de la atención estándar para mejorar los resultados postoperatorios en pacientes que se someten a una cirugía de reemplazo de cadera o de rodilla, relacionado al dolor, al funcionamiento y a la duración de la estancia hospitalaria. Hay pruebas de que la educación prequirúrgica tiene efecto beneficioso sobre la ansiedad prequirúrgica y esto hace que los pacientes se adaptan con mayor facilidad a los cambios que se presenten en el posoperatorio.

Otro de los estudios a encontrados fue el de Atik y col ${ }^{(11)}$ en Mallorca España quienes realizan una investigación con el título de "Estandarización de los cuidados de enfermería en Preanestesia", como resultado elaboran un manual de enfermería con plan de cuidados durante el pre, intra y postoperatorio; centrándose en cubrir sólo las necesidades físicas, preparación prequirúrgica, procedimientos como profilaxis antibiótica, uso adecuado de prendas quirúrgicas, técnica de venopunción; entre otras quedando fuera lo psicológico.

A nivel nacional el estudio de Cárdenas Andrea ${ }^{(12)}$ relacionado con la histerectomía y ansiedad, mediante un estudio comparativo entre dos tipos de preparación psicológica prequirúrgica que buscó comparar dos tipos de preparación para cirugía en mujeres programadas para histerectomía abdominal. El primer grupo, de 30 mujeres, recibió 
una preparación basada en la consideración particular de las estrategias de afrontamiento. El segundo grupo, también de 30 mujeres, recibió preparación mediante información escrita. Como variables de resultado se estudiaron los niveles de ansiedad pre y posoperatoria, la magnitud del dolor postoperatorio percibido y la presencia de complicaciones posquirúrgicas inmediatas. Los resultados revelaron que las participantes del grupo que recibió el primer tipo de programa presentaron mayor disminución en la ansiedad, menor nivel de dolor percibido y una baja frecuencia de complicaciones, que aquellas que recibieron la preparación a través de información escrita.

Otro estudio a nivel Nacional ${ }^{(13)}$ es el relacionado con ansiedad y cirugía, mediante un estudio de tipo observacional descriptivo transversal, que busca evaluar el nivel de ansiedad (cognitiva y somática) de los pacientes sometidos a cirugía programada, identificando los distintos agentes estresores existentes previos a la cirugía, para realizar una intervención preventiva, por medio de un protocolo de actuación consistente en aportar información por escrito de la intervención quirúrgica y de la anestesia, aportando apoyo psicológico de acuerdo a la su necesidad del paciente. Consideran de notable importancia reorientar y/o crear protocolos de actuación prequirúrgica incidiendo en el aspecto psicológico y formativo del sujeto antes de la intervención, proporcionándole información del proceso al que va a ser sometido, brindando apoyo psicológico y técnicas de afrontamiento, psicoprofilaxis quirúrgica, solventándole dudas e inquietudes, para mejorar la calidad de los cuidados que se le proporciona a los pacientes, proporcionando al profesional de Enfermería un documento escrito para ponerlo en práctica, teniéndo en cuenta los límites propios del marco teórico-práctico de la profesión.

De igual manera creen que esta preparación tendrá efectos beneficiosos para el sujeto en el período quirúrgico, especialmente durante la intervención (anestesia) y concretamente en el postoperatorio tanto inmediato como diferido, disminuyendo los tiempos de estancia en los mismos, así como la reducción en la administración de fármacos (analgésicos, hipnóticos) y de hospitalización; por lo cual se debe realizar la visita preoperatoria para así disminuir los síntomas desagradables en el posoperatorio inmediato como lo afirma Echeverry Gustavo y col en la Universidad del Valle ${ }^{(14)}$, establecen que, independiente del proceso quirúrgico, esta situación ocasiona al paciente y su familia una serie de alteraciones en su comportamiento que pueden ser aliviadas con la preparación emocional al paciente y a su familia, con una explicación clara sobre el procedimiento que se le va a practicar y las condiciones en que se encontrará después de la cirugía. De lo anterior se concluye que la preparación sicológica del paciente y su familia en esta etapa preoperatoria, ayuda a reducir los problemas y la ansiedad que le causa la proximidad de la intervención quirúrgica.

Por lo tanto, el cuidado de enfermería debe tener en cuenta la educación a los pacientes que serán sometidos a algún procedimiento quirúrgico como se evidencia en el estudio de Chemes de Fuentes) ${ }^{(15)}$ donde instruye al paciente en la fase preoperatoria como las técnicas de respiraciones profundas, a toser en forma eficaz $u$ otro ejercicio que la cirugía exija. Los ejercicios pasivos y activos de miembros inferiores mejoran la circulación y previenen el éxtasis venoso que favorece la 
recuperación, reduce las complicaciones y el período de hospitalización. Lo que hace que disminuyan las respuestas fisiológicas que incluyen la estructura, anatómica y genéticas.

Mediante las intervenciones de enfermería se fortalece el nivel de conocimiento del paciente en pro de su bienestar físico como lo afirma el estudio de Walter $\mathrm{D}^{(16)}{ }_{2}$ donde las funciones de las enfermeras están fundamentadas científicamente, en el proceso de atención de enfermería, con el fin de cubrir las necesidades del paciente y sus familiares disminuyendo así los factores de riesgo afectivos y cognitivos, que puede impactar las experiencias de los síntomas, incluyendo el grado de incertidumbre del individuo sobre la enfermedad o el síntoma para evitar complicaciones después de la intervención quirúrgica, por ello debe personalizar el cuidado de enfermería de acuerdo a las características individuales del paciente.

Mediante esta revisión bibliográfica se encontró que existe una necesidad de establecer intervenciones de enfermería orientadas al abordaje integral de la vivencia de los pacientes en los procesos quirúrgicos; en la teoría de rango medio de síntomas desagradables, Elizabeth R.Lenz y Lida C. Pugh ${ }^{(17)}$ propone mejorar la experiencia de la sintomatología en varios contextos, el brindar información útil para diseñar métodos efectivos de prevención y mejorar sus efectos negativos. Teniendo en cuenta los tres componentes de la teoría: los síntomas, factores influyentes y resultados de desempeño.

Se han encontrado estudios que validan la importancia de la teoría de los síntomas desagradables, como es el estudio de Espinoza Maritza y col (18), ellos enfatizaron en los factores influyentes, los síntomas y las consecuencias de las experiencias de los síntomas, donde se estructuró una guía de atención en oncología realizada por el Ministerio de Salud chileno, la Norma de Alivio del Dolor y Cuidados Paliativos ${ }^{(5)}$ basada en las recomendaciones de la OMS, que orienta el cuidado y control de síntomas en personas con cáncer. Sus protocolos de cuidados, están basados en evidencia científica imperante a nivel nacional y mundial, para la práctica del cuidado de enfermería. Orientando a la estructuración de guías o protocolos de cuidados propios de enfermería. Donde esta teoría estimula un pensamiento integrador frente al abordaje y manejo de las manifestaciones, los cuales hacen que el cuidado de enfermería cumpla con los tres principios que son eficacia, eficiencia y efectividad en la atención de los servicios, como lo demuestra el estudio de Salazar Ángela María $^{(19)}$ en el área quirúrgica, quien hace énfasis en los cuidados de enfermería junto con el equipo quirúrgico para dar atención en los enfoques de valoración, satisfacción de las necesidades y expectativas del paciente, por el riesgo que implica el procedimiento anestésico y quirúrgico.

Desde su desarrollo y conceptualización, la teoría ha formado parte del marco teórico, en un estudio de McCann y Boore ${ }^{(20)}$ de fatiga realizado a 39 pacientes adultos de enfermedad renal en etapa final recibiendo hemodiálisis. Los resultados sugirieron que los factores físicos como la edad, género, estado físico, y medidas bioquímicas de diálisis adecuada, hemoglobina, hematocrito, creatinina, albumina fosfato, y calcio, factores psicológicos como la ansiedad y depresión estos dos factores actúan juntos y recíprocamente para influenciar la intensidad de fatiga. Sin embargo, factores situacionales función del rol, estado marital, estado de empleado fueron no relativos a la fatiga. Ellos concluyeron que "la fatiga está influenciada por factores psicológicos y sociológicos, los cuales a su vez tienen relaciones reciprocas uno con el otro" La 
fatiga impacto el desempeño, como se indica por el rol físico, nivel de actividad y motivación.

La teoría se aplica en diferentes situaciones Parshall y Cols ${ }^{(17)}$, realizaron una investigación con pacientes con disnea y otros síntomas, similarmente Pugh y Milligan ${ }^{(17)}$ realizaron una investigación dirigida a los factores psicológico, sociológico y situacional en pacientes que estaban en lactancia materna. En lo que concluyen que esta teoría permite a enfermería estructurar guías o protocolos de cuidados propios de enfermería, por su enfoque integrador frente al abordaje y manejo de las manifestaciones.

\section{CONCLUSIONES}

- La atención de enfermería en los pacientes que van a ser sometidos a un proceso quirúrgico requiere un conocimiento profundo y que mediante la intervención de enfermería en la dimensión cognitiva y física pueden disminuir los efectos de los síntomas como lo indican los diferentes estudios revisados.

- Los pacientes que esperan una intervención quirúrgica suelen experimentar distintos miedos y esperanzas. El personal de enfermería debe determinar las percepciones, emociones, conductas y sistemas de apoyo que pueden ayudar a superar los efectos manifestados.

- El hecho de que el individuo afronte la cirugía con altos niveles de ansiedad puede tener influencias negativas en la recuperación físico-psíquica, postquirúrgica, tales como largas estancias hospitalarias o mayor necesidad de analgésicos, lo cual supone un perjuicio tanto para el individuo como par el sistema de salud por su elevado coste económico.

- Las teorías de mediano rango analizan aspectos vividos del ejercicio profesional y de las características únicas de la práctica de la enfermería, dentro de un contexto mucho más objetivo. Permiten que la enfermera y el enfermero realicen un análisis integral de los síntomas y su impacto en los entornos social, cultural y emocional, y pueda establecer intervenciones de cuidado individuales y no generalizadas.

\section{REFERENCIAS BIBLIOGRÁFICAS}

1. United States Preventive Task Force (USPSTF). [Citado 2007 diciembre 18];

Disponible en: http://www.ahrq.gov

2. Acuña P; Ingrid del Pilar. Grado de ansiedad preoperatoria en pacientes que van a ser intervenidos en el Hospital Nacional.Perú.2009.(Citado el 13 de Febrero de 2012). Disponible en: http://ateneo.unmsm.edu.pe/ateneo/bitstream/123456789/2569/1/Acu\%C3\%B1a Polo Ingrid del Pilar 2009.pdf.

3. Chirveches E; Arnau A; Soley M; Rosell F; Clotet G; Roura P; Oriol M; Isern O; Faro M.Efecto de una visita prequirúrgica de enfermería perioperatoria sobre la ansiedad y 
el dolor. Barcelona. España. Febrero de 2006. (Citado el 22 de febrero de 2012). Disponible en: <http://www.elsevier.es/es/revistas/enfermeria-clinica-35/efecto-unavisita-prequirurgica-enfermeria-perioperatoria-ansiedad-13084268-originales-2006>.

4. Pérez Díaz R; Martín C, Marta C., Quiñones Castro M. Influencia de la ansiedad prequirúrgica en la evolución de la cirugía de las várices. Rev. Cubana Angiol y CirVasc 2000;1(2):95-100. (Citado el 9 de febrero del 2012). Disponible en: <http://bvs.sld.cu/revistas/ang/vol1_2_00/ang02200.htm>

5. Chavez Meza N. Efectividad de un programa educativo en el incremento del nivel de conocimiento que tiene el paciente pre-quirúrgico en el Hospital II Es Salud Suarez Angamos. Lima-Perú. Febrero de 2009.

6. Achury Saldaña DM. Aplicación de la teoría de los síntomas desagradables en el manejo del dolor. Investigación en Enfermería: Imagen y Desarrollo, vol. 9, núm. 2, junio-diciembre, 2007,p. 8-19. Pontificia Universidad Javeriana -Colombia. (Citado el 14 de marzo del 2012). Disponible en http://redalyc.uaemex.mx/pdf/1452/145212858002.pdf

7. Berendina E; Bouwman C; Siqueira CD. Cuidados de enfermería realizados en el paciente quirúrgico en el período preoperatorio, Rev. esc. enferm. USP vol.43 no.1 São Paulo, Marzo 2009. (Citado el 15 de febrero del 2012). Disponible en: <http://dx.doi.org/10.1590/S0080-62342009000100002>.

8. Priego Álvarez $\mathrm{H}$ y col. Calidad de la visita preoperatoria de la enfermera quirúrgica.2008.

9. Lavinas Santos y col. Comunicación terapéutica en la atención pre-operatoria de mastectomía. 2010.Revista Brasileira de Enfermagem. Print versión ISSN00347167.Rev. bras. enferm.Vol.63No.4BrasiliaJuly/Aug.2010. (Citado el 15 de febrero del 2012). Disponible en internet http://dx.doi.org/10.1590/S0034-71672010000400027

10. McDonald S, Hetrick S, Green S. Educación prequirúrgica para el reemplazo de cadera o de rodilla - Australuia(Revisión Cochrane traducida). En: La Biblioteca Cochrane Plus, 2008 Número 4. Oxford: Update Software Ltd. Disponible en: <http://www.update-software.com.> (Traducida de The Cochrane Library, 2008 Issue 3. Chichester, UK: John Wiley\&Sons, Ltd.). (Citado el 14 de Marzo del 2012).

11. Atik PA, Pinillos BM. Gestión y Evaluación de Costes Sanitarios - Estandarización de los cuidados de enfermería en Preanestesia, Vol. 3 - Número 4 - Octubrediciembre 2002. (Citado el 20 de febrero del 2012) Disponible en:<http://hispasante.hispagenda.com/documentacion/guias/enfermeria/Estandarizaci on_cuidados_enfermeria_Preanestesia.pdf>. Fecha de acceso: 20, feb. 2012.

12. Cárdenas A; Quiroga C; Restrepo M; Cortés D. Histerectomía y ansiedad: estudio comparativo entre dos tipos de preparación psicológica prequirúrgica. Revista Colombiana de Obstetricia y Ginecología, vol. 56, núm. 3, 2005, pp. 209-215. Federación Colombiana de Asociaciones de Obstetricia y Ginecología Colombia. (Citado el 13 de febrero del 2012). Disponible en: <http://redalyc.uaemex.mx/redalyc/pdf/1952/195214311003.pdf

13. Barrilero Gómez JA., Casero Mayorga JA. Y col. .Ansiedad y Cirugía. Repercusiones en el paciente sometido a cirugía. Diplomado en Enfermería (1996) (Citado el 9 de febrero del 2012). Disponible en:<http://www.uclm.es/ab/enfermeria/revista/numero\%208/ansicirug.htm>Fecha de acceso:9 de Feb. del 2012

14. Echeverry G, y col. Universidad del Valle Cuidados peri operatorios del paciente quirúrgico. Dirección de Educación Desescolarizada. 2002. (Citado el 13 de febrero del 2012). Disponible en internet $h$ ttp://www.objetos.univalle.edu.co/?q=node/368 15. Chemes de Fuentes C. Tucumán - Argentina Año 2008. (Citado el 20 de febrero del 2012).

Disponible en internet: 
<http://www.fm.unt.edu.ar/carreras/webenfermeria/documentos/Enfermeria_Quirurgica Modulo_01.pdf>

16. Walter D. Cuidados de enfermería a usuarios hospitalizados de la Unidad Clínica de Cirugía en la fase preoperatoria del Hospital Uyapar de Puerto Ordaz Estado Bolívar. 30 septiembre 2011. (Citado el 20 de febrero del 2012). Disponible en internet:<http://www.saber.ucv.ve/123456789/369>

17. Lenz y col. Teoría de los síntomas desagradables. 2000

18. Espinoza Venegas M y col. Universidad de Concepción, Chile. Análisis de la Teoría de los Síntomas Desagradables en el Cuidado de la Enfermería Paliativa Oncológica. (Citado el 20 de febrero del 2012). Disponible en internet:<http://bvs.sld.cu/revistas/enf/vol27_02_11/enf06211.htm <

19. Salazar AM. Cirugía: Un Contexto Diferente De Cuidado. Artículo de Investigación. 2011. (Citado el 20 de febrero del 2012). Disponible en internet http://www.enfermeria.unal.edu.co/revista/articulos/xxix1 6.pdf

20. McCann K, Boore JRP. Fatigue in persons with renal failure who require maintenance haemodialysis. J Adv Nurs. 2000 (cited 2012 Marzo 14) : 32 (5): 1132142. Available from: http://www.ncbi.nlm.nih.gov/pubmed/11114998 